\title{
INCIDENCE OF CARBAPENEM RESISTANT NONFERMENTING GRAM NEGATIVE BACILLI FROM PATIENTS WITH RESPIRATORY INFECTIONS IN THE INTENSIVE CARE UNITS
}

\author{
P Gladstone, P Rajendran, *KN Brahmadathan
}

\begin{abstract}
Resistance to carbapenems is commonly seen in nonfermenting gram negative bacilli (NFGNB). We document herein the prevalence of carbapenem resistance in NFGNB isolated from patients with respiratory tract infections in the intensive care units (ICUs). A total of 460 NFGNB were isolated from 606 endotracheal aspirate specimens during January through December 2003, of which $56(12.2 \%)$ were found to be resistant to imipenem and meropenem. Of these, $24(42.8 \%)$ were Pseudomonas aeruginosa, 8 (14.2\%) were Acinetobacter spp. and 24 (42.8\%) were other NFGNB. Stringent protocols such as antibiotic policies and resistance surveillance programs are mandatory to curb these bacteria in ICU settings.
\end{abstract}

Key words: Carbapenem resistance, $N F G N B, I C U s$

Carbapenems first introduced in 1980 are now frequently used as the last choice in treating serious infections caused by multidrug resistant gram negative bacilli. These antibiotics are stable to $\beta$-lactamases including the extended spectrum $\beta$ lactamases (ESBLs) and AmpC produced by gram negative bacilli. Unfortunately resistance to these antibiotics started emerging from 1990 and has been reported in nonfermenting gram negative bacilli (NFGNB) worldwide over the years with varying frequencies. ${ }^{1-3} \mathrm{NFGNB}$, normally a saprophyte, cause serious infections in immunocompromised and hospitalized patients especially those admitted to intensive care units (ICU). ${ }^{4}$ These organisms further worsen the situation by virtue of their

multidrug resistance and thus limit therapeutic options. ${ }^{4}$ Pseudomonas aeruginosa and Acinetobacter spp. in particular are most often associated with carbapenem resistance. This is of significance since NFGNB can cause fatal lower respiratory tract infections in patients admitted to ICU. Unfortunately, there is paucity of data on the prevalence of carbapenem resistance in the Indian literature. ${ }^{5}$ Here, we document the microbiological aspects of the prevalence of carbapenem resistance in NFGNB isolated from patients with respiratory tract infections in the ICU.

\section{Materials and Methods}

A total of 606 endotracheal aspirates from suction tips of patients on ventilators with clinical evidence of lower respiratory tract infections were processed for bacterial pathogens, in a semi quantitative manner. These specimens

*Corresponding author (email: <knb1948@ hotmail.com>) Department of Clinical Microbiology, Christian Medical College, Vellore - 632 004, Tamil Nadu, India.

Received: 13-12-2004

Accepted: 27-4-2005 were from patients admitted to medical (MICU), surgical (SICU), neuro (NICU) and pediatric (PICU) intensive care units at Christian Medical College, Vellore, between January and December 2003. The specimens were cultured and the bacterial isolates were identified by methods standardized in our laboratory. ${ }^{6}$ Antibiotic susceptibility testing for the nonfastidious aerobic gram negative bacilli was carried out on Mueller-Hinton agar by the Kirby Bauer Disc diffusion method using ampicillin $(10 \mu \mathrm{g})$, cefotaxime $(30 \mu \mathrm{g})$, ceftazidime $(30 \mu \mathrm{g})$, cefpirome $(30 \mu \mathrm{g})$, imipenem $(10 \mu \mathrm{g})$, meropenem $(10 \mu \mathrm{g})$, gentamicin $(10 \mu \mathrm{g})$, amikacin $(30 \mu \mathrm{g})$ and isepamicin $(30 \mu \mathrm{g})$ discs and interpreted according to NCCLS guidelines. ${ }^{7}$ ATCC control strains were used for every round of testing.

\section{Results}

A total of 732 gram negative bacilli were isolated from 606 endotracheal aspirate specimens. This included NFGNB (460), Escherichia coli (80), Klebsiella spp. (119), Enterobacter spp. (39), Citrobacter spp. (13), Proteus mirabilis (10), Morganella morganii (5), Aeromonas spp. (4), Serratia spp. (1), and Salmonella typhi (1). Fifty six (12.2\%) of the 460 NFGNB were found to be resistant to imipenem and meropenem. Of these, 24 (42.8\%) were Pseudomonas aeruginosa, $8(14.2 \%)$ were Acinetobacter spp. and 24 $(42.8 \%)$ were other NFGNB. All strains were also resistant to the other seven antibiotics tested. Gram negative bacilli other than NFGNB were all susceptible to the two carbapenems.

\section{Discussion}

This study documents a carbapenem resistance of $12.2 \%$ among 460 NFGNB isolated from ICU patients on ventilator over a one-year period. It shows a considerably higher prevalence of resistance among Ps.aeruginosa (42.8\%) than 
Acinetobacter spp. (14.2\%). All isolates were resistant to both imipenem and meropenem. Similar data are not available for comparison in the Indian literature; however Taneja et $a l^{5}$ reported that $36.4 \%$ of the nonfermenters $(n=85)$ causing nosocomial urinary tract infections were resistant to imipenem. In another study, Navaneeth et $a l^{8}$ reported a prevalence of $12 \%$ carbapenem resistance among 50 strains of Pseudomonas aeruginosa isolated from various clinical specimens. Despite the low prevalence of carbapenem resistance in our study, caution has to be exercised in its usage in ventilated patients to arrest any further increase in the resistance to carbapenems.

This is a retrospective analysis and the data was generated from our routine diagnostic laboratory where many strains were not preserved for further speciation. Despite this, we believe that documenting carbapenem resistant NFGNB is very important as these strains may often cause outbreaks in the ICU setting, cause increased morbidity and mortality in patients with underlying disease, or limit therapeutic options due to the high degree of multidrug resistance. This means that these organisms are potentially dangerous in the ICU setting and can lead to increased financial burden for the patients. They may also spread resistance to other susceptible bacteria by horizontal gene transfer. To the best of our knowledge, this is the first report from India on carbapenem resistance in NFGNB from respiratory secretions of patients in the ICUs.

Reportedly, several outbreaks due to carbapenem resistant NFGNB have resulted with considerable morbidity and mortality. ${ }^{9,10}$ Resistance to these antibiotics is due to decreased outer membrane permeability, increased efflux systems, alteration of penicillin binding proteins and carbapenem hydrolyzing enzymes - carbapenemases. These carbapenemases may be class B-metallo $\beta$-lactamases (IMP, VIM) or class D-oxacillinases (OXA-23 to OXA-27) or class A- clavulanic acid inhibitory enzymes (SME, NMC, IMI, KPC). ${ }^{11}$ They may be chromosomally or plasmid mediated and therefore pose a threat of spread of resistance by gene transfer among gram negative bacteria.

Since carbapenem resistance is mediated by several mechanisms, cross resistance is commonly seen among related antibiotics. Although there are various specific tests ${ }^{11,13}$ to detect the underlying mechanism of carbapenem resistance, Kirby-Bauer disc diffusion test is a simple, easy to perform and cost effective test which can be conveniently used to screen carbapenem resistance. These strains also remain resistant to several other antibiotics including penicillins, cephalosporins, quinolones, amino glycosides and third generation cephalosporins including ceftazidime and cefotaxime. Thus, they may be ESBL producers as well. In this study, all 56 strains were resistant to all antibiotics tested so that there was no alternative antibiotic that could be used to treat infections caused by them. This poses serious problems in choosing the right antibiotic for the treatment of sick patients admitted into the ICU. This disturbing situation could be attributed to the increased use of antibiotics which has to be controlled by strict antibiotic policy. Various strategies such as strict infection control measures, judicious prescribing of antibiotics, antibiotic resistance surveillance programs and antibiotic cycling have all been tried. ${ }^{12}$ Regular monitoring and documentation of carbapenem resistance is therefore crucial in developing strategies to control infections due to these bacteria in patients admitted to ICU.

\section{References}

1. Tognim MC, Andrade SS, Silbert S, Gales AC, Jones RN, Sader HS. Resistance trends of Acinetobacter spp. in Latin America and characterization of international dissemination of multi-drug resistant strains: five-year report of the SENTRY Antimicrobial Surveillance Program. Int J Infect Dis.2004;8:284-91

2. Deshpande LM, Fritsche TR, Jones RN. Molecular epidemiology of selected multidrug-resistant bacteria: a global report from the SENTRY Antimicrobial Surveillance Program. Diagn Microbiol Infect Dis. 2004;49:231-6.

3. Ouinn PJ. Clinical problems posed by multiresistant Nonfermenting gram-negative pathogens. Clin Infect Dis 1998;27:117-24

4. Goossens H. Susceptibility of multi-drug-resistant Pseudomonas aeruginosa in intensive care units: results from the European MYSTIC study group. Clin Microbiol Infec. 2003;9:980-3.

5. Taneja N, Aharwal S M, Sharma M. Imipenem resistance in nonfermentors causing nosocomial urinary tract infections. Indian J Med Sci.2003;57:294-9.

6. Myers. R M, Koshi. G. Manual of Diagnostic procedures in Medical Microbiology and Immunology / Serology (All India Press, Pondicherry) 1982. p. 50-2

7. National Committee for Clinical Laboratory Standards. Performance standards for antimicrobial susceptibility testing. Twelfth informational supplement. M100-S12 NCCLS, 2002. Wayne P.A.

8. Navaneeth BV, Sridaran D, Sahay D, Belwadi MR. A preliminary study on metallo-beta-lactamase producing Pseudomonas aeruginosa in hospitalized patients. Indian J Med Res.2002;116:264-7.

9. Corbella X, Montero A, Pujol M, et al. Emergence and rapid spread of carbapenem resistance during a large and sustained hospital outbreak of multiresistant Acinetobacter baumannii. $J$ Clin Microbiol 2000;38:4086-5.

10. Landman D, Quale JM, Mayorga D, Adedeji A, Vangala K, Ravishankar J, et al Citywide clonal outbreak of multiresistant Acinetobacter baumannii and Pseudomonas aeruginosa in Brooklyn, NY: the preantibiotic era has returned. Arch Intern Med 2002;162:1515-20.

11. Yu YS, Yang Q, Xu XW, Kong HS, Xu GY, Zhong BY. Typing and characterization of Carbapenem resistant Acinetobacter calcoaceticus - baumannii complex in a Chinese hospital. J Med 
Microbiol 2004;53:653-6.

12. Merz LR, Warren DK, Kollef MH, Fraser VJ. Effects of an antibiotic cycling program on antibiotic prescribing practices in an intensive care unit. Antimicrob Agents Chemother 2004;48:2861-5.
13. Lee K, Lim YS, Yong D, Yum JH, Chong Y. Evaluation of the Hodge test and the imipenem-EDTA double-disk synergy test for differentiating metallo-beta-lactamase-producing isolates of Pseudomonas spp. and Acinetobacter spp. J Clin Microbiol 2003;41:4623-9.

\section{Attention Subscribers}

The subscriptions for Indian Journal of Medical Microbiology are being serviced from the following address:

Medknow Publications

A-108/109, Kanara Business Center

Off Link Rd, Ghatkopar (E)

Mumbai - 400075, INDIA

Phone: $91-22-56491818$

Fax: 91-22-5649 1817

The subscription rates of the journal are as follows:

\begin{tabular}{cc}
\multicolumn{1}{c}{ One year (4 } & issues) \\
Indian & Rs. 1500 \\
Overseas & $\$ 150$
\end{tabular}

Personal subscriptions paid from personal funds and Institutional subscription are available at $5 \%$ discounted rates.

Please send all renewals and new subscriptions along with the payment to the above address. Cheques/Demand Draft should be made payable to Medknow Publications. Please add Rs.20/for outstation cheques.

If you wish to receive the Journal by registered post, please add Rs.60/- per annum to the total payment and make the request at the time of subscribing. 